\title{
Underwater Glider Motion Control Based on Neural Network
}

\author{
Kedong LYU \\ Department of Automation Engineering, Tianjin Electronic Information Technician College, \\ Tianjin, China
}

\begin{abstract}
Underwater glider is an important equipment for ocean research, water quality detection and other ocean missions. It needs very high precision requirements to meet underwater glider motion control. When the position of buoyancy system changes, the balance parameters will change significantly. This paper presents a method for calculating the balance parameters of underwater glider based on neural network. In order to verify the effectiveness of the neural network control, the South China Sea experiment was carried out. By comparing the analysis results with the actual situation, the experiment shows that the neural network model is feasible.
\end{abstract}

Keywords: Underwater Glider, Neural Network, Balance Parameters

\section{Introduction}

With the rapid development of marine bionic robots, more and more robots are used in ocean exploration and ocean monitoring. The underwater glider can accomplish various tasks and has various functions. At the same time, the underwater glider has the adaptive ability to various complex environments. In recent years, the rapid development of underwater vehicles has been applied to more and more challenging underwater missions. The underwater glider becomes a low-cost autonomous underwater glider by changing the position of the buoyancy system ${ }^{[1-4]}$. When the position of buoyancy system changes, the equilibrium parameters will change critically. Balance parameters are very important for motion control and glider flight trajectory. Under complex sea conditions, the equilibrium parameters are not constants, but a series of dynamic numbers. Usually, the equilibrium parameters are obtained through a large number of experiments to find out a series of feasible parameters. Although this method is simple, it has great uncertainty, especially when the environment changes. This method is not suitable for complex underwater environment.

In this paper, a new motion control method of underwater glider based on neural network with balanced parameters is proposed. The purpose of this study is to find the most suitable balance parameters to optimize the balance effect of glider and further improve the control effect of glider navigation. In the mathematical model of PETREL Glider, the estimation of hydrodynamics and the analysis of motion control are carried out. In this paper, Newton method is used to model the motion of glider under the action of current. In addition, the strip theory and computational fluid dynamics (CFD) are used to estimate the hydrodynamics. Considering the balance state and the influence of wave and current, a motion control model of PETREL Glider based on neural network is proposed. The dynamics of Underwater Gliders in the ocean can be described more accurately. According to the equilibrium parameters and CFD fluid dynamics, the dynamic equation is established by using neural

\footnotetext{
* Corresponding author. Tel.: +86-131-32270395; fax: +86-022-88241604

E-mail address: scut_08@sina.com

Manuscript History:

Received 7 April, 2019, Revised 29 September, 2019, Accepted 29 September, 2019, Published 30 September, 2019
}

e-ISSN: 2289-7771

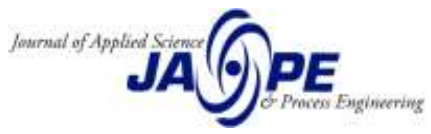


network predictive control (NNPC), and the state equation is rewritten. It can better adapt to different marine environments.

\section{PETREL Glider}

Combining with hydrodynamics and mechanism theory, UG structure, performance and various control parameters are optimized to enhance the ability of glider to detect various complex marine environments and select operation strategies independently. A new type of PETREL Glider is designed. The glider consists of a cylindrical hull, two symmetrical wings, two rudders, a sealed bunker, a turbo generator, an electromagnetic generator, a pervious tank and a control and drive mechanism. PETREL Glider has higher working efficiency and better observation accuracy, which further improves the endurance of underwater glider, and provides insistent basis for long-range, large-scale ocean observation technology and low-speed accurate observation in small waters. Table 1 presents the principal characteristics of the glider, while in Figure 1, presents the reference frames of the glider.

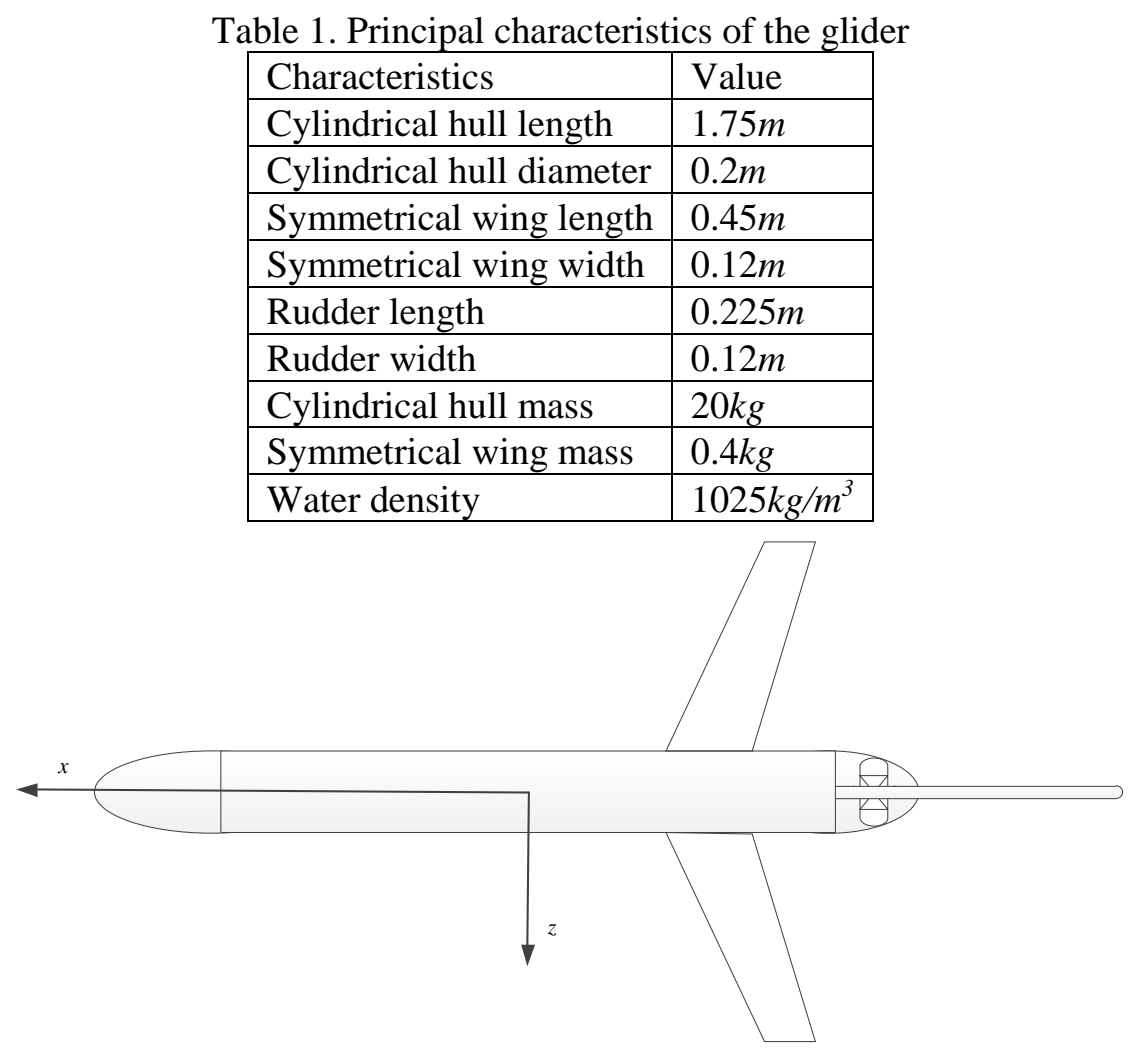

Figure 1. Reference frames of the glider

\section{Instruments}

Through the characteristics of strong modeling ability of neural network for underwater glider, the dimension size of data needed for prediction model is effectively reduced, and the value of data is really excavated. In this paper, the balance parameters of underwater glider are analyzed, and the practical problems such as sea water fluctuation are combined. By comparing with other traditional methods, it is proved that the neural network has higher accuracy and applicability ${ }^{[6-8]}$.

\subsection{Pitch adjusting tests}


Two groups or more of pitch adjusting tests are held in the pool which imitates the sea condition. The more tests we do, the more precision. The pitch adjusting test contains two steps: Step I is moving the position of battery pack, step II is moving of the position of oil mass.. When diving or rising, the underwater glider changes buoyancy by changing the amount of oil inside and outside the oil sac. The state of glider is chang at the same time. The purpose of the test is to find the relationship of the battery position, oil mass and the pitching angle.

\subsection{Extract data and data preprocessing}

Data preprocessing refers to some processing of data before the main processing. It is necessary to eliminate the impact of a large lag system. Two sets of data were taken from the water glider adjustment test. According to the analysis, the position of the battery pack and the amount of oil as an input factor are suitable for the prediction model, and the output factor is the pitch angle. One set of data is used as training data and another set of data is used as test data.

\subsection{Second-order headings}

The network model including five parts, they respectively are the input layer and input nodes, the hidden layer and hidden nodes, the output layer and output nodes, the transfer function used in the PETREL Glider and the training methods based on the neural network. So, in the PETREL Glider, the most significant aspect of the decision in the neural network model development is to choose how many hidden nodes to be used in PETREL Glider. Compared with ordinary neural networks, the biggest difference of PETREL Glider neural network is that each hidden layer unit is not independent. Each hidden layer is not only related to each other, but also related to the timing input before the time it receives. This feature is very helpful for processing time-related data. This article analyzed the change trend and the experience formula under different ocean conditions in a large number of experiments, and choose equation (1).

$$
l=(m+n)^{1 / 2}+\alpha
$$

Where, $l$ is the number of hidden layer nodes, $\mathrm{m}$ is the number of input layer nodes, $n$ is the number of output layer nodes, and $\alpha=1,2 \ldots . .10$.

As shown in this experiment of the PETREL Glider neural network model of hidden layer network, two hidden layer network is much better than one hidden layer network. The best hidden nodes is $l_{1}=8$, and $l_{2}=6$. As shown in Figure 2 below.

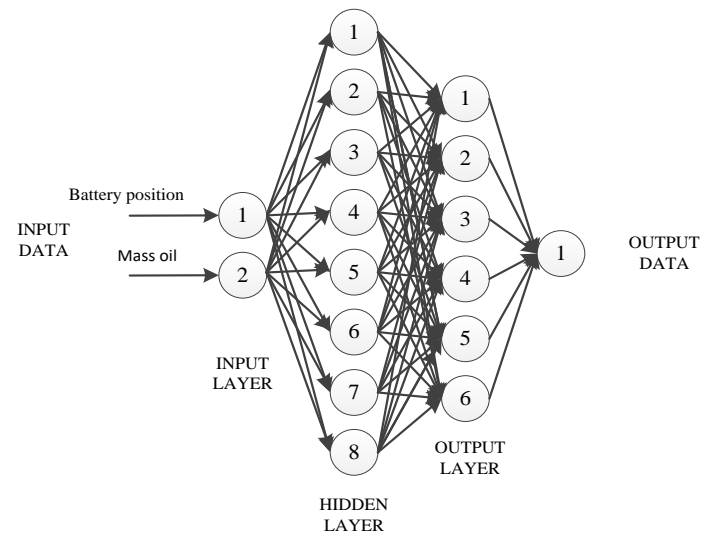

Figure 2. Neural network model 


\section{Hydrodynamic model}

\subsection{Hydrodynamic parameters}

The movement of the water glider is easily affected by the irregular force of the ocean current. When it is disturbed by the outside world, the irregular movement state experienced due to the uneven force and the acceleration and deceleration phases appear. The severity hydrodynamic directly determines the outcome of the movement is good or bad. In the vertical plane movement, hydrodynamic mainly lift Resistance and trim moment. According to the references [9-11], equation (2) (3) (4) can be shown as below.

$$
\begin{aligned}
& L=\frac{1}{2} \rho C_{L}(\alpha) A V^{2}=\left(K_{L 0}+K_{L} \alpha\right)\left(v_{1}^{2}+v_{3}^{2}\right) \\
& D=\frac{1}{2} \rho C_{D}(\alpha) A V^{2}=\left(K_{D 0}+K_{D} \alpha^{2}\right)\left(v_{1}^{2}+v_{3}^{2}\right) \\
& M_{D L}=\frac{1}{2} \rho C_{M}(\alpha) A V^{2}=\left(K_{M 0}+K_{M} \alpha\right)\left(v_{1}^{2}+v_{3}^{2}\right)
\end{aligned}
$$

Where, $\rho$ is the density of sea water, $A$ is the cross sectional area of glider, $V$ is the resultant velocity of glider, $v_{l}$ and $v_{3}$ respectively are the horizontal speed component and vertical speed component of glider, $\alpha$ is angle of attack, $C_{L}(\alpha), C_{D}(\alpha), C_{M}(\alpha)$ respectively are the parameters of hydrodynamic model.

\subsection{Propeller thrust}

According to the references [9-11], equation (5) can be shown as below.

$$
T=K_{T} \rho d_{p}^{4} n^{2}
$$

Where, $T$ is the propeller thrust of the glider, $K_{T}$ is the coefficient of the glider, $\rho$ is the density of sea water, $d$ is the diameter of the glider, $n$ is the rotate speed of propeller.

\subsection{Dynamic model of underwater glider}

According to the stress analysis, using the Lagrangian method is to set up the dynamic equation of the glider in vertical plane. Equation (6)-(15) can be shown as below.

$$
\begin{gathered}
\dot{\Omega}_{2}=v_{1} \cos \theta+v_{3} \sin \theta \\
\dot{z}=-v_{1} \sin \theta+v_{3} \cos \theta \\
\dot{\theta}=\Omega_{2} \\
\left.\dot{\Omega}_{2}\left(m_{3}-m_{1}\right) v_{1} v_{3}-\left(r_{P 1} P_{P 1}+r_{P 3} P_{P 3}\right) \Omega_{2}-\bar{m} g\left(r_{P 1} \cos \theta+r_{P 3} \sin \theta\right)+M_{D L}-r_{P 3} u_{1}+r_{P 1} u_{3}\right] \\
\dot{v}_{1}=\frac{1}{m_{1}}\left(-m_{3} v_{3} \Omega_{2}-P_{P 3} \Omega_{2}-m_{0} g \sin \theta+L \sin \alpha-D \cos \alpha-u_{1}+T\right) \\
\dot{v}_{3}=\frac{1}{m_{3}}\left(m_{1} v_{1} \Omega_{2}+P_{P 1} \Omega_{2}-m_{0} g \cos \theta-L \cos \alpha-D \sin \alpha-u_{3}\right) \\
\dot{r}_{P 1}=\frac{1}{m_{p}} P_{P 1}-v_{1}-r_{P 3} \Omega_{2}
\end{gathered}
$$




$$
\begin{aligned}
& \dot{r}_{P 3}=\frac{1}{m_{p}} P_{P 3}-v_{3}-r_{P 1} \Omega_{2} \\
& \dot{P}_{P 1}=u_{1} \\
& \dot{P}_{P 3}=u_{3}
\end{aligned}
$$

Where, $r_{p l}, r_{p 3}$ respectively are the position of sliding mass in the $x$ and $z$ direction, $p_{p l}, p_{p 3}$ respectively are the force of sliding mass in the $x$ and $z$ direction, $m_{p}$ is the internal sliding mass, $m_{1}, m_{3}$ respectively are the hull mass in the $x$ and $z$ direction.

\section{Experiments}

According to the neural network model build insection 4, select the parameters, battery position and oil mass, as the equilibrium state. Balance parameters are important for motion control and glider's path. In the complex sea conditions, balance parameters are not constant numbers, but these are a series of dynamic numbers. Balance parameters are obtained through the neural network model build in section 4 to find a series of feasible parameters. This neural network model method is suitable for the complex underwater environment.

As shown in Figure 3, iterative learning can get the parameters of battery position and the oil mass position. As shown in Figure 4, the output of glider system and model output used NN method is same with the tolerable error. As shown in Figure 5, control signal and system output can meet the needs.
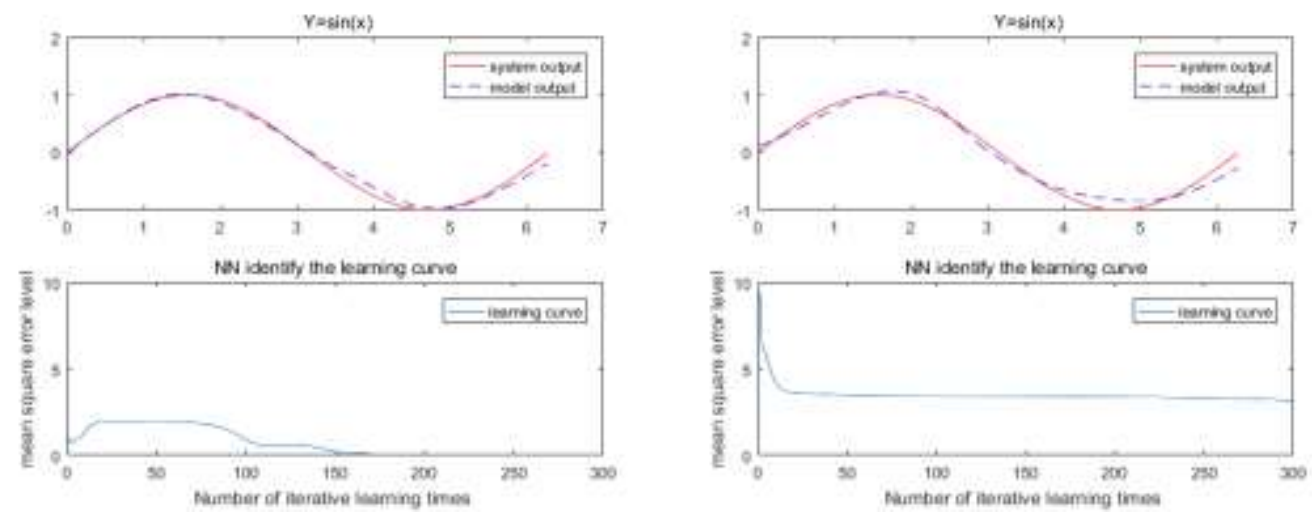

Figure 3. Number of iterative learning times

Figure 3 shows the number of iterative learning times. From Figure 3, it can be seen that the number of learning curve exceeds 300 , and the mean square error level is greatly reduced. 

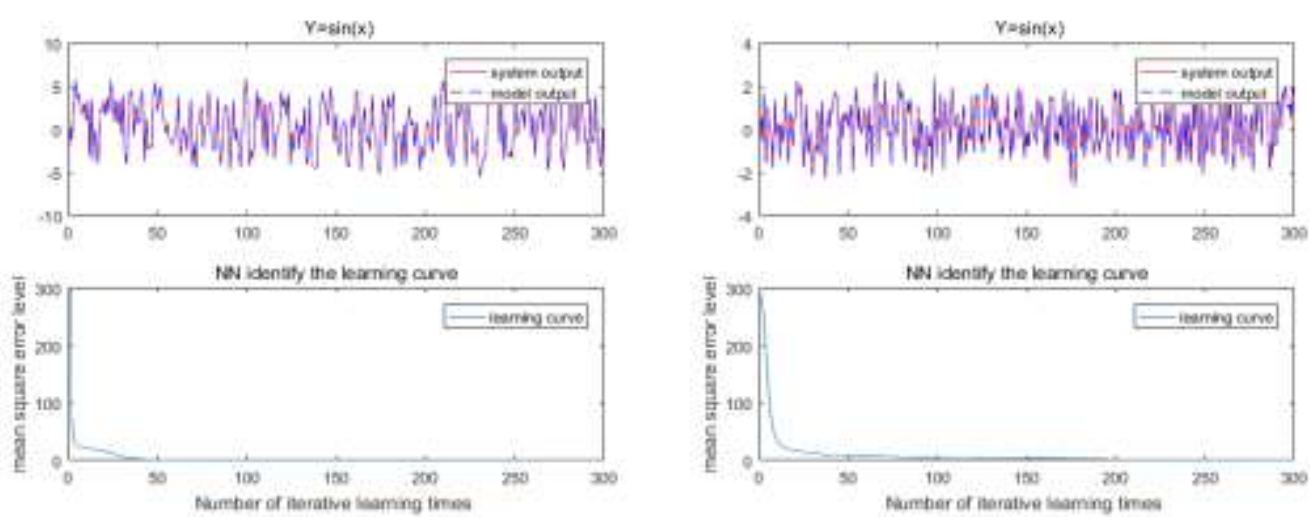

Figure 4. The output of glider system and model output used NN method

Figure 4 shows the output of glider system and model output used NN method. From Figure 4, it can be seen that the number of learning curve exceeds 300 , and the mean square error level is greatly reduced.
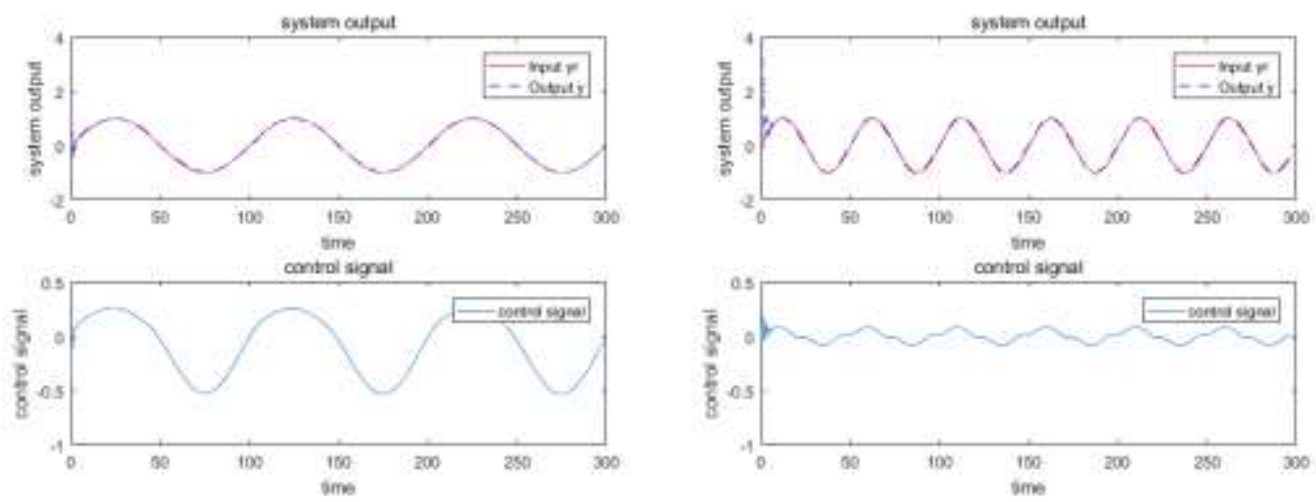

Figure 5. Control signal and system output

Figure 5 shows the control signal and system output. From Figure 5, it can be seen that the number of learning curve exceeds 300 , and the mean square error level is greatly reduced.In order to verify the effectiveness of the neural network method, experiments were conducted in the South China Sea. PETREL Glider used the neural network model began to glide and complete the task well according to the given tasks. After a number of tests, the error of the PETREL Glider's water discharge position and the set position is within 5\% during a gliding cycle. Under the traditional method, the error of the PETREL Glider's water discharge position and set position is about 10\%. Compared with the traditional method, neural network has a significant improvement.

\section{Conclusions}

Mechanical structure according to PETREL Glider, through pitch adjusting tests, extract data and data preprocessing and second-order headings, select the neural network method, build the Hydrodynamic model, and test in the South China Sea. Underwater glider motion control based on neural network is an effective system and it can reduce the error of the PETREL Glider's water discharge position and the set position from $10 \%$ to 5\%. By comparing the analysis results with the actual situation, the experiment shows that the neural network model is feasible. 


\section{References}

[1] Javaid, M. Y., Ovinis, M. , Hashim, F. B. M. , Maimun, A. , Ahmed, Y. M. , and Ullah, B. . (2016). Effect of wing form on the hydrodynamic characteristics and dynamic stability of an underwater glider. International Journal of Naval Architecture and Ocean Engineering, S2092678216304691.

[2] Ma, Z. , Wang, Y. , Wang, S. , and Yang, Y. . (2016). Ocean thermal energy harvesting with phase change material for underwater glider. Applied Energy, 178, 557-566.

[3] Yang, Y. , Wang, Y. , Ma, Z. , and Wang, S. . (2016). A thermal engine for underwater glider driven by ocean thermal energy. Applied Thermal Engineering, S1359431116000934.

[4] J. FalcãO Carneiro, J. , and Gomes, d. A. F. . (2016). Model of a thermal driven volumetric pump for energy harvesting in an underwater glider. Energy, 112, 28-42.

[5] Fan, S. S., Yang, C. J., Peng, S. L., Kai-Hu, L. I., Xie, Y., and Zhang, S. Y. (2013). Underwater glider design based on dynamic model analysis and prototype development. Journal of Zhejiang Universityence C, 14(8), 583-599.

[6] Ningl, Y. , Liu, Y. , and Ji, Q. . (2010). Bayesian - BP Neural Network based Short-Term Load Forecasting for power system. International Conference on Advanced Computer Theory \& Engineering. IEEE.

[7] Ma, S. , and Guan, L. . (2011). Arc-Fault Recognition Based on BP Neural Network. Third International Conference on Measuring Technology \& Mechatronics Automation. IEEE Computer Society.

[8] Wen, J. W. , Chen, C. , and Yan, X. C. . (2012). Based on BP neural network forecast bridge temperature field and its effect on the behavior of bridge deflection. International Conference on Transportation. IEEE.

[9] Kennel, C. F., and Coroniti, F. V. (1984). Magnetohydrodynamic model of crab nebula radiation. Astrophysical Journal, 283(2), 710-730.

[10] Gardner, C. L. (2007). The quantum hydrodynamic model for semiconductor devices. Siam Journal on Applied Mathematics, 54(2), 409-427.

[11] North, E. W., Adams, E. E., Schlag, Z., Sherwood, C. R., He, R., and Hyun, K. H., et al. (2011). Simulating oil droplet dispersal from the deepwater horizon spill with a lagrangian approach. Geophysical Monograph, $195,217-226$. 